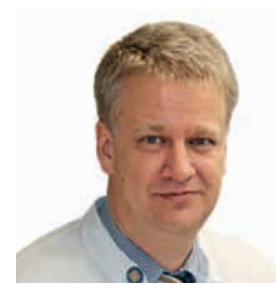

Prof. Dr. Eggert Stockfleth eggert.stockfleth@charite.de

\title{
Ein überzeugendes Konzept für den Wissenstransfer in den Praxisalltag
}

Kaum ein medizinisches Fach macht so schnelle Fortschritte wie die Dermatologie. Dieses liegt sicherlich auch daran, dass das Organ Haut so gut einsehbar und damit für wissenschaftliche Fragestellungen gut erreichbar ist. Gerade in den letzten Jahren sehen wir uns mit einer Flut von neuen, praxisrelevanten Informationen konfrontiert, die nicht immer leicht verständlich sind und in ihrer Fülle an neuem Wissen sehr zeitintensiv im Studium sind.

Die neue Zeitschrift Karger Kompass DermaTOLoGie hat den Anspruch, Entwicklungen aus der internationalen Forschung in den Praxisalltag der Ärzte zu transportieren. Die national und international anerkannten Experten selektieren und kommentieren praxisrelevante Originalartikel aus Karger-Zeitschriften und komprimieren so die Inhalte. Alles wird in deutscher Sprache publiziert, was das Lesen und das Verstehen zusätzlich erleichtert.

In der ersten Auflage, die Ihnen hier vorliegt, gibt es unterschiedliche, hochinteressante Themenschwerpunkte. Zunächst gibt es eine Übersichtsarbeit mit dem Titel «Antimikrobielle Peptide und die Haut: Ein Paradigma der translationalen Medizin» - eine Thematik, die in den letzten Jahren immer mehr Beachtung gewonnen hat. Im Bereich «Wissenstransfer» werden Themen von grundsätzlicher Bedeutung diskutiert, die un- sere Disziplin insgesamt betreffen. So befasst sich das erste Heft Karger Kompass Dermatologie mit der konfokalen Mikroskopie, dem Hautkrebs-Screening, der Corticosteroid-Pulstherapie bei Alopecia areata, der Psoriasis und Kontaktdermatitis, den Körperpflegeprodukten, Condylomata acuminata, der Wundreinigung und vielem anderen mehr - allesamt Themen, die derzeit (zum Teil auch wieder) hochaktuell sind. Der Abschluss dieses neuen Heftes beschäftigt sich mit «Erfahrungen aus der Praxis». Hier werden interessante Krankheitsentitäten wie das PASH-Syndrom nach einer Darm-Bypass-Operation und Haarverlust nach einer Herpes-Zoster-Behandlung praxisnah besprochen. Insgesamt möchte ich allen Autoren und dem Karger Verlag mit seinem gesamten Team gratulieren zu diesem sehr überzeugenden neuen Konzept zum Wissenstransfer aus Dermatology, Skin Pharmacology and Physiology und Case Reports in DermaTOLOGY.

Ich bin mir sicher, dass der Karger Kompass Dermatologie zukünftig ein guter Wegweiser für die Dermatologinnen und Dermatologen im deutschsprachigen Raum sein wird, und wünsche den Verantwortlichen für die Zukunft alles nur erdenklich Gute.

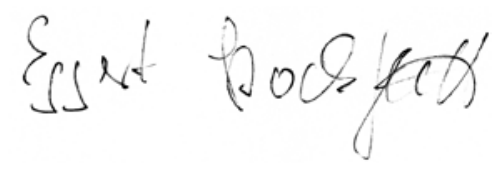

\section{KARGER \\ Fax +497614520714 \\ information@karger.com} www.karger.com
(C) 2013 S. Karger GmbH, Freiburg

$2296-5424 / 13 / 0011-0009 \$ 38.00 / 0$

Accessible online at:

www.karger.com $/ \mathrm{kkd}$
Prof. Dr. Eggert Stockfleth

Klinik für Dermatologie, Venerologie und Allergologie

Charité - Universitätsmedizin Berlin, Campus Mitte

Charitéplatz 1, 10117 Berlin (Deutschland) 